\title{
The Gyromagnetic Factor of Elementary Particles and Non-Minimal Coupling
}

Jefferson Morais*

CBPF - Centro Brasileiro de Pesquisas Físicas, Brazil

E-mail: moraisecbpf.br

Antônio Accioly

Instituto de Física Teórica - UNESP, Brazil

CBPF - Centro Brasileiro de Pesquisas Físicas, Brazil

E-mail: accioly.antoniodgmail.com

José Abdala Helayël-Neto

CBPF - Centro Brasileiro de Pesquisas Físicas, Brazil

E-mail: helayelecbpf.br

\section{Rodrigo Turcati}

CBPF - Centro Brasileiro de Pesquisas Físicas, Brazil

E-mail: turcati@cbpf.br

In this work we investigate a possible magnetic moment generation for massive neutral particles with spins-1 and -2 coupled non-minimally, in a specific way, to an external electromagnetic field. It is found that, in the nonrelativistic limit, these particles present $g=1$. This result, worked out in the framework of Relativistic Quantum Mechanics, seems to suggest that $g=1$ for all massive and neutral particles of any spin $\leq 2$. We also compare with the results obtained for massive charged particles of spins- 1 and -2 , in the same regime (nonrelativistic), in order to investigate the role played by the spin separetely from the charge.

5th International School on Field Theory and Gravitation, April 20 - 242009

Cuiabá city, Brazil

\footnotetext{
*Postersection.
} 


\section{Introduction}

The spin can be responsible for some important effects. An interesting area arised from some aspects due to the spin, called spintronic, is showing the important role played by the spin of charged particles [1]. As accelerators get improved, we became able to investigate more and more properties of elementary particles. So, a natural question is the influence of the spin in electromagnetic interaction of this kind of particles, especcialy for the neutral ones. Actually, magnetic properties of neutral particles have been reasonably studied in the literature, specially for neutrinos in the context of Salam-Weinberg-Glashow theory [2] [3] [乌] [5].

We know that, classicaly, a particle of mass $M$ and charge $Q$ moving with velocity $\vec{v}$ in a closed loop is equivalent to a magnetic dipole of moment $\vec{\mu}$, wich is proportional to the angular moment $\vec{L}$, being the proportionality factor equal to $\frac{Q}{2 M}$. Extending the definition of $\vec{\mu}$, we can write

$$
\vec{\mu}=g \frac{Q}{2 M} \vec{L},
$$

where $g$ is the gyromagnetic factor. The introduction of the spin- $\frac{1}{2}$ for the electron represented a conflic between the classical result $(g=1)$ and the real value $g=2$ for this elementary particle. This was explained by the Dirac's theory of electron. For some decades, based on the fact that the natural coupling for the interaction between elementary particles is the minimal coupling - i.e. the replacement of the ordinary derivative by the gauge covariant one - physicists believed in the general result

$$
g=\frac{1}{s}
$$

where $s$ is the spin of the particle, for charged truly-elementary particles [6] [7] [8]. This was another goal of Dirac's theory. Unfortunatelly, when applied for the vector bosons $\mathbf{W}$ - the only known charged spin-1 trully-elementary particle up to now - it got fail, since $g=2$ at tree level for these bosons. Interestingly, making use of general properties of the S-Matrix, Weinberg [10] had shown in the begining of the seventies that $g \approx 2$, for massive charged elementary particles with arbitrary spin. About two decades after, Ferrara, Porrati and Telegdi [11] obtained the same result by using non-minimal electromagnetic coupling in the Lagrangian.

Assuming the importance of non-minimal coupling in explaining the correct gyromagnetic factor for charged particles, we propose, in this work, an extension of the electromagnetic interaction to neutral particles. Our main objective is analyse, in the quantum mechanics level, how elementar particle of spin- 1 or -2 can acquire a magnetic moment, even the particle being neutral, by using non-minimal coupling. The procedure adopted is, starting from appropriate relativistic wave equations for each case, we study the nonrelativistic regime, in the presence of a non-minimal coupling.

\section{Massive Vector Boson}

We begin our discussion by the vector bosons $W^{ \pm}$. As said before, the only known massive charged particle with spin-1, up to now. This case had been already studied in the literature, representing, therefore, a good test of the mechanism adopted through all this work. Our starting point 
is the Lagrangian density

$$
\mathscr{L}=-\frac{1}{2} W_{\mu \nu}^{*} W^{\mu \nu}+m^{2} W_{\mu}^{*} W^{\mu}+e e F_{\mu \nu} W^{* \mu} W^{v},
$$

where $W_{\mu \nu}=D_{\mu} W_{v}-D_{v} W_{\mu}, D_{\mu}=\partial_{\mu}+\imath e A_{\mu}$ and $W^{* \mu}$ is the charge conjugate of $W^{\mu}$. Note the non-minimal term in the equation above. It is easy to show that considering just minimal coupling results in the wrong $g$ for these bosons. The variation with respect to $W^{* \mu}$ produces the field equation

$$
D_{\mu} W^{\mu v}+m^{2} W^{v}+\imath e F^{v \mu} W_{\mu}=0 .
$$

From this relation, we obtain for the components of $W^{\mu}$, in the nonrelativistic limit $(E \cong m)$ :

$$
W^{0}=\frac{\vec{p}}{m} \cdot \vec{W}-\frac{e}{m} \vec{A} \cdot \vec{W}
$$

and

$$
E_{N R} W_{i}=\frac{1}{2 m}(\vec{p}-e \vec{A})^{2} W_{i}-\vec{\mu}_{i j} \cdot \vec{B} W_{j}
$$

where $E_{N R}$ is the nonrelativistic energy of the particle and

$$
\vec{\mu}_{i j}=\frac{e}{m} \vec{S}_{i j}
$$

the magnetic moment of the particle, with $\left(S_{k}\right)_{i j} \equiv-\imath \varepsilon_{k i j}$ being the spin matrix. From the first equation one can see that the time component is proportional to the spatial component, with a very small proportionality factor $\left(\sim \frac{v}{c}\right)$ in the low energy limit. The Pauli-type equation for the propagating component of the field reveals the importance of non-minimal coupling in producing the correct value $g=2$ for the bosons $\mathbf{W}$.

Now we can study the case of neutral vector bosons. Our proposal is

$$
\partial_{\mu} Z^{\mu v}+m^{2} Z^{v}+\frac{\lambda}{2} F^{\kappa \lambda}\left(\Sigma_{\kappa \lambda}\right)^{v \mu} Z_{\mu}=0
$$

that represents the addition of a non-minimal term taking into account the spin of the particle in the Proca description of the field $Z^{\mu}$. The $\Sigma_{\kappa}$ 's stand for the generators of the Lorentz group in the vector representation. So, the term in $\lambda$ is describing the coupling between the spin of the neutral vector boson to the external electromagnetic field. Taking the matrix elements of $\Sigma_{\kappa \lambda}$ as given below,

$$
\left(\Sigma_{\kappa \lambda}\right)^{v \mu}=\delta_{\kappa}^{v} \delta_{\lambda}^{\mu}-\delta_{\kappa}^{\mu} \delta_{\lambda}^{v}
$$

the field equation becames

$$
\partial_{\mu} Z^{\mu v}+m^{2} Z^{v}+\lambda F^{v \mu} Z_{\mu}=0
$$

As in the case of $W^{\mu}$, it is easy to show that the time component of $Z^{\mu}$ is of order $\left(\frac{v}{c}\right)$ of its spatial component. So, performing the nonrelativistic approximation on the field equation for the propagating component, results

$$
E_{N R} Z_{i}=\frac{\vec{p}^{2}}{2 m} Z_{i}+\frac{\lambda}{2 m} \vec{S}_{i j} \cdot \vec{B} Z_{j}=0
$$

where $\left(S_{k}\right)_{i j} \equiv-\varepsilon_{k i j}$. A direct check reveals that $\lambda$ has charge dimension. It indicates that $g=$ 1. This interesting result is showing that the boson $\mathbf{Z}$ can have a eletromagnetic interaction due exclusively to its spin, assuming that non-minimal coupling is allowed. 


\section{Massive Spin-2 Field}

The expectation of massive graviton production at LHC and their decays following a pattern of jets sets out a very interesting perspective in conection with the Brane World scenario [12] [13] [14] . In this context, a good point may be the analysis of the possible photon-graviton coupling, in order to obtain enough information about the intrinsic properties of the gravitons, such as a possible magnetic moment due only to their spin. Recently, Porrati and Rahman have proposed a mechanism for studying these possible interaction [15]; these authors treat the charged spin-2 case, since they are basically interested in consistency aspects of higher spins propagation. Here we will do the same analysis performed in the previous section. Like we did for the neutral boson $\mathbf{Z}$, we propose a photon-graviton interaction by the addition of a non-minimal term in the Pauli-Fierz field equation that describe a massive neutral spin-2 particle:

$$
\begin{aligned}
& \square h_{\mu v}-\partial_{\mu} \partial^{\alpha} h_{v \alpha}-\partial_{v} \partial^{\alpha} h_{\mu \alpha}+\partial_{\mu} \partial_{v} h+m^{2}\left(h_{\mu v}+\frac{1}{2} \eta_{\mu v} h\right)+ \\
& +\frac{\lambda}{2} F^{\kappa \lambda}\left(\Sigma_{\kappa \lambda}\right)_{\mu}^{\alpha} h_{\alpha v}+\frac{\lambda}{2} F^{\kappa \lambda}\left(\Sigma_{\kappa \lambda}\right)_{v}^{\alpha} h_{\alpha \mu}=0,
\end{aligned}
$$

where

$$
\left(\Sigma_{\kappa \lambda}\right)_{\mu}^{\alpha}=\eta_{\kappa \mu} \delta_{\lambda}^{\alpha}-\eta_{\lambda \mu} \delta_{\kappa}^{\alpha} .
$$

Again the terms in $\lambda$ account for the coupling between the spin of the particle and the external electromagnetic field.

After some algebraic steps, one can show that the only propagating component is the purely spatial and traceless $h_{i j}=h_{j i}$, holding, in this way, the spin-2 propagation (five independent components). In the nonrelativistic approximation, the equation for this propagating component reads:

$$
E_{N R} h_{i j}=\frac{\vec{p}^{2}}{2 m} h_{i j}+\frac{\lambda}{2 m} \vec{B} \cdot\left(\vec{S}_{i k} h_{j}^{k}+\vec{S}_{j k} h_{i}^{k}\right)
$$

with $\left(S_{l}\right)_{i k}=-\varepsilon_{l i k}$.

As in the $Z^{\mu}$ case, here the coupling constant $\lambda$ also has charge dimension, wich indicates, again, the result $g=1$ in the low energy level. This magnetic interaction arised only due to the graviton spin, since the field $h^{\mu v}$ is a neutral field. In order to compare, we shall do the same procedure for the massive charged spin-2 particle. Our starting point is the Lagrangian density

$$
\begin{aligned}
\mathscr{L}= & -\frac{1}{4}\left[D_{\lambda} h^{* \mu v} D^{\lambda} h_{\mu v}-2 D_{\mu} h^{* \mu v} D^{\alpha} h_{\alpha v}+D_{\mu} h^{* \mu v} D_{v} h+D_{\mu} h^{\mu v} D_{v} h^{*}-\right. \\
& \left.D_{\mu} h^{*} D^{\mu} h-m^{2}\left(h_{\mu v}^{*} h^{\mu v}-h^{*} h\right)\right]+\imath e F^{\mu v} h_{\mu \rho}^{*} h_{v}^{\rho},
\end{aligned}
$$

We said in the $W^{\mu}$ case that the consideration of a minimal coupling alone results in the wrong value for the gyromagnetic factor of this particle. Here, only the minimal prescription for the interaction between the field and the charged spin-2 particle is not enough to produce a magnetic moment term. That is the reason we start considering the non-minimal $\imath e F^{\mu v} h_{\mu \rho}^{*} h^{\rho}{ }_{v}$ term, that [15] calls a dipole term. A variation with respect to $h^{* \mu v}$ produces

$$
\begin{aligned}
& \widetilde{\square} h_{\mu v}-D_{\mu} D^{\alpha} h_{\alpha v}-D_{v} D^{\alpha} h_{\alpha \mu}+\frac{1}{2}\left(D_{\mu} D_{v}+D_{v} D_{\mu}\right) h+ \\
& +m^{2}\left(h_{\mu v}+\frac{1}{2} \eta_{\mu v} h\right)+2 u e\left(F_{\mu}^{\rho} h_{v \rho}+F_{v}^{\rho} h_{\mu \rho}\right)=0,
\end{aligned}
$$


with $\widetilde{\square} \equiv D^{\alpha} D_{\alpha}$. Again one can show that the propagating component is the purely spatial and traceless $h_{i j}=h_{j i}$, for wich the Pauli-type equation in the nonrelativistic limit reads:

$$
E_{N R} h_{i j}=\frac{1}{2 m}(\vec{p}-e \vec{A})^{2} h_{i j}+\left(\vec{\mu}_{i k} h_{j}^{k}+\vec{\mu}_{j k} h_{i}^{k}\right) \cdot \vec{B}
$$

with the magnetic dipole moment identified by

$$
\vec{\mu}_{i j} \equiv \frac{e}{m} \vec{S}_{i j}
$$

Note the importance of the non-minimal term in this case. It generates a magnetic interaction with the correct gyromagnetic factor, $g=2$, as expected for these classes of particles.

\section{Conclusion}

In this contribution, our efforts have been to point out the importance of non-minimal electromagnetic coupling. This kind of interaction is consistent with the propagation of the spin considered in any case, produces the correct gyromagnetic factor for charged massive particles and opens up a possibility of extending the electromagnetic interaction for neutral particles, since we observed a magnetic moment generation due only to the spin of the particle. As a future work, we shall investigate if $g=1$ is a fundamental result for neutral elementary particles with any spin, in contrast to the result $g=2$ for charged particles. Another subject we are interested in is the case of a massive graviton in scenarios with Lorentz-Symmetry breaking [16] [17]. Such an activity has been pushed forward in conection with efective theories derived from Strings. It would be interesting to compute the contribution from the Lorentz-Symmetry breaking background to the magnetic moment of neutral spin-2 particles.

\section{Acknowledgments}

Jefferson Morais would like to thank CAPES/CBPF for partial finantial support and the organizing committee of the 5th International School on Field Theory and Gravitation for partial finantial support and the hospitality during the event.

\section{References}

[1] A. Vernes, B.L. Györffy, P. Weinberger, Spin currents, spin-transfer torque, and spin-Hall effects in relativistic quantum mechanics, Physical Review B76, 012408 (2007).

[2] Y. Aharonov and A. Casher, Topological Quantum Effects for Neutral Particles Phys. Rev. Lett. 53:319 (1984).

[3] H. Belich, L.P. Colatto, T. Costa-Soares, J.A. Helayel-Neto, and M.T.D. Orlando, Magnetic Moment Generation from non-minimal couplings in a scenario with Lorentz-Symmetry Violation, Eur. Phys. J. C62:425-432 (2009).

[4] Nicole F. Bell, Vincenzo Cirigliano, Michael J. Ramsey-Musolf, Petr Vogel, and Mark B. Wise,How magnetic is the Dirac neutrino?, Phys. Rev. Lett. 95:151802 (2005).

[5] Barry R. Holstein, How large is the 'natural' magnetic moment?, Am. J. Phys. 74:1104-1111 (2006). 
[6] F.J. Belifante, Intrinsic Magnetic Moment of Elementary Particles of Spin $\frac{3}{2}$, Phys. Rev. 92:997-1001 (1953).

[7] K.M. Case, Phys. Rev. 94,1442 (Z6) (1954).

[8] C. Fronsdal, Nuovo Cimento Suppl. 9, 416 (1958).

[9] J. Schwinger, Particles, Sources and Fields, Addison-Wesley, Reading, MA, 1970.

[10] S. Weinberg, Lectures on Elementary Particles and Quantum Field Theory, Proceedings of the Summer Institute, Brandeis University (1970), edited by S. Deser, MIT Press, Cambridge, MA, 1970, Vol. I.

[11] S. Ferrara, M. Porrati, V. Telegdi, Phys.Rev. D46:3529-3537 (1992).

[12] Gouranga C. Nayak. Graviton and radion production at LHC: From $p$ p and Pb Pb collisions. [hep-ph/0211395] (2002).

[13] Qiang Li, Chong Sheng Li, and Li Lin Yang. Soft gluon resummation effects in single graviton production at the CERN Large Hadron Collider in the Randall-Sundrum model, Phys. Rev. D74:056002 (2006).

[14] Kaoru Hagiwara, Partha Konar, Qiang Li, Kentarou Mawatari, and Dieter Zeppenfeld, Graviton production with 2 jets at the LHC in large extra dimensions, JHEP 04:019 (2008).

[15] Massimo Porrati and Rakibur Rahman. Electromagnetically Interacting Massive Spin-2 Field: Intrinsic Cutoff and Pathologies in External Fields. [hep-th/0809.2807] (2008).

[16] V.A. Kostelecky and S. Samuel, Phys. Rev. D39, 683 (1989).

[17] H. Belich, T. Costa-Soares, M.A. Santos e M.T.D. Orlando,Revista Brasleira de Ensino de Física, V. 29, n. 1, p. 57-64 (2007). 